\title{
EEG-Based Emotion Recognition Using Frequency Domain Features and Support Vector Machines
}

\author{
Xiao-Wei Wang ${ }^{1}$, Dan $\mathrm{Nie}^{1}$, and Bao-Liang $\mathrm{Lu}^{1,2, \star}$ \\ 1 Center for Brain-Like Computing and Machine Intelligence \\ Department of Computer Science and Engineering \\ 2 MOE-Microsoft Key Lab. for Intelligent Computing and Intelligent Systems \\ Shanghai Jiao Tong University \\ 800 Dong Chuan Road, Shanghai 200240, China \\ bllu@sjtu.edu.cn
}

\begin{abstract}
Information about the emotional state of users has become more and more important in human-machine interaction and braincomputer interface. This paper introduces an emotion recognition system based on electroencephalogram (EEG) signals. Experiments using movie elicitation are designed for acquiring subject's EEG signals to classify four emotion states, joy, relax, sad, and fear. After pre-processing the EEG signals, we investigate various kinds of EEG features to build an emotion recognition system. To evaluate classification performance, $k$ nearest neighbor $(k \mathrm{NN})$ algorithm, multilayer perceptron and support vector machines are used as classifiers. Further, a minimum redundancymaximum relevance method is used for extracting common critical features across subjects. Experimental results indicate that an average test accuracy of $66.51 \%$ for classifying four emotion states can be obtained by using frequency domain features and support vector machines.
\end{abstract}

Keywords: human-machine interaction, brain-computer interface, emotion recognition, electroencephalogram.

\section{Introduction}

Emotion plays an important role in human-human interaction. Considering the proliferation of machines in our commonness, emotion interactions between humans and machines has been one of the most important issues in advanced human-machine interaction (HMI) and brain-computer interface (BCI) today [1]. To make this collaboration more efficient in both HMI and BCI, we need to equip machines with the means to interpret and understand human emotions without the input of a user's translated intention.

Numerous studies on engineering approaches to automatic emotion recognition have been performed. They can be categorized into two kinds of approaches.

\footnotetext{
^ Corresponding author.
} 
The first kind of approaches focuses on the analysis of facial expressions or speech [2] 3. These audio-visual based techniques allow noncontact detection of emotion, so they do not give the subject any discomfort. However, these techniques might be more prone to deception, and the parameters easily vary in different situations. The second kind of approaches focuses on physiological signals, which change according to exciting emotions and can be observed on changes of autonomic nervous system in the periphery, such as electrocardiogram (ECG), skin conductance (SC), respiration, pulse and so on 45. As comparison with audiovisual based methods, the responses of physiological signals tend to provide more detailed and complex information as an indicator for estimating emotional states.

In addition to periphery physiological signals, electroencephalograph (EEG) captured from the brain in central nervous system has also been proved providing informative characteristics in responses to the emotional states [6]. Since Davidson et al. 7] suggested that frontal brain electrical activity was associated with the experience of positive and negative emotions, the studies of associations between EEG signals and emotions have been received much attention.

So far, researchers often use two different methods to model emotions. One approach is to organize emotion as a set of diverse and discrete emotions. In this model, there is a set of emotions which are more basic than others, and these basic emotions can be seen as prototypes from which other emotions are derived. Another way is to use multiple dimensions or scales to categorize emotions. A two dimensional model of emotion is introduced by Davidson et al. 8]. According to this model, emotions are specified by their positions in the two-dimensional space as shown in Figure 1, which is spanned by two axes, valence axis and arousal axis. The valence axis represents the quality of an emotion ranging from unpleasant to pleasant. The arousal axis refers to the quantitative activation level ranging from calm to excited. The different emotional labels can be plotted at various positions on a $2 \mathrm{D}$ plane spanned by these two axes.

Since emotional state corresponds to a separate subsystem in the brain, EEG signals can reveal important information on their functioning. The studies of associations between EEG activity and emotions have been received much attention. Bos used the international affective picture system (IAPS) and international affective digitized sound system (IADS) for eliciting emotional states [9]. They achieved an average classification accuracy of $65 \%$ for arousal and $68 \%$ for valance by using alpha power and beta power as features and fisher's discriminant analysis (FDA) as classifiers. Takahashiet et al. used EEG signal to recognize emotion in response to movie scenes [10]. They achieved a recognition rate of $41.7 \%$ for five emotion states. In our previous work, we proposed an emotion recognition system using the power spectrum of EEG as features [1]. Our experimental results indicated that the recognition rate using a support vector machine reached an accuracy of $87.5 \%$ for two emotion states .

Despite much efforts have been devoted to emotion recognition based on EEG in the literature, further research is needed in order to find more effective feature extraction and classification methods to improve recognition performance. In this paper, we deal with all of the essential stages of EEG-based emotion recognition 


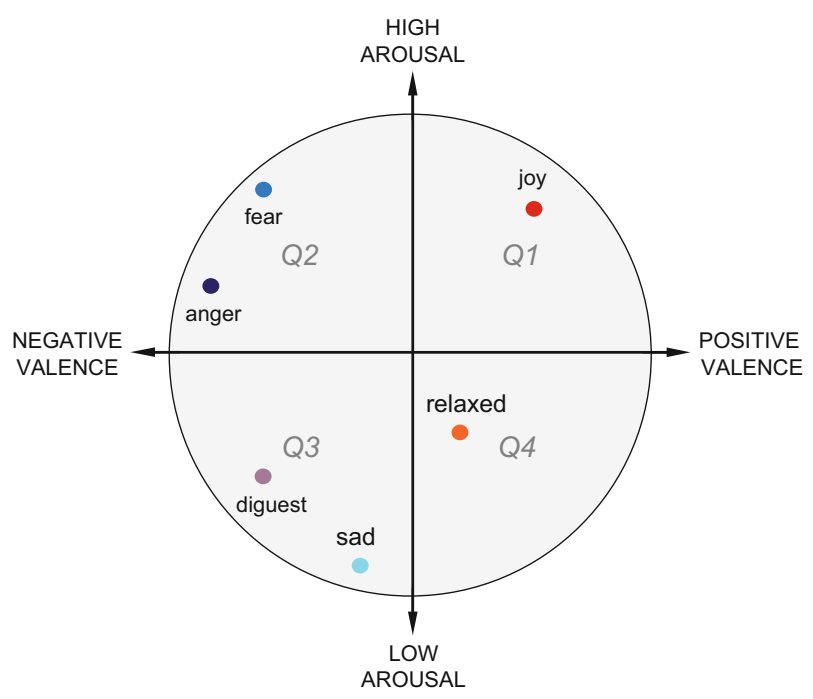

Fig. 1. Two-dimensional emotion model

systems, from data collection to feature extraction and emotion classification. Our study has two main purposes. The first goal is to search emotion-specific features of EEG signals, and the second goal is to evaluate the efficiency of different classifiers for EEG-based emotion recognition. To this end, a user-independent emotion recognition system for classification of four typical emotions is introduced.

\section{Experiment Procedure}

\subsection{Stimulus Material and Presentation}

To stimulate subject's emotions, we used several movie clips that were extracted from Oscars films as elicitors. Each set of clips includes three clips for each of the four target emotions: joy (intense-pleasant), relax (calm-pleasant), sad (calm-unpleasant), and fear (intense-unpleasant). The selection criteria for movie clips are as follows: a) the length of the scene should be relatively short; b) the scene is to be understood without explanation; and c) the scene should elicit single desired target emotion in subjects and not multiple emotion. To evaluate whether the movie clips excite each emotion or not, we carried out investigation using questionnaires by human subjects who don't take part in the experiment to verify the efficacy of these elicitors before the experiment.

\subsection{Participants}

Five right-handed health volunteers (two males, three females), 18-25 years of age $($ mean $=22.3$ and $\mathrm{SD}=1.34)$, participated in the study. All subjects had no per- 


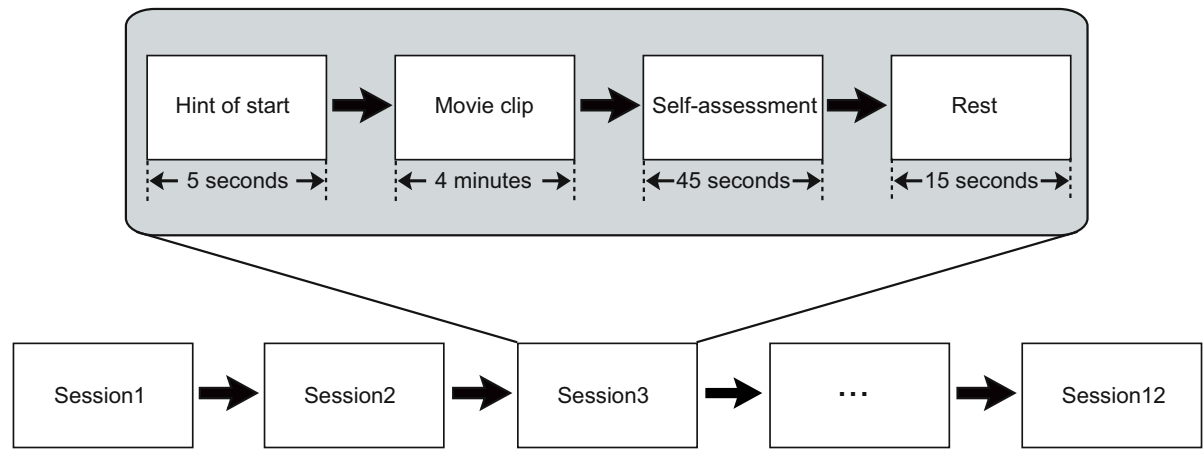

Fig. 2. The process of experiment

sonal history of neurological of psychiatric illness and had normal or correctednormal vision. All subjects were informed the scope and design of the study.

\subsection{Task}

In order to get quality data, subjects were instructed to keep their eyes open and view each movie clip for its entire duration in the experiment. Movie clips inducing different emotion conditions were presented in a random order. Each movie clip was presented for 4 to 5 minutes, preceded by $5 \mathrm{~s}$ of blank screen as the hint of start. At the end of each clip, subjects were asked to assign valence and arousal ratings and to rate the specific emotions they had experienced during movie viewing. The rating procedure lasted about 45 seconds. An inter trial interval $(15 \mathrm{~s})$ of blank screen lapsed between movie presentations for emotion recovery. Valence and arousal ratings were obtained using the Self-Assessment Manikin (SAM) 12. Four basic emotional states, joy, relax, sad, and fear, describing the reaction to the movie clips were also evaluated at the same time. The given self-reported emotional states were used to verify EEG-based emotion classification.

\subsection{EEG Recording}

A 128-channel electrical signal imaging system (ESI-128, NeuroScan Labs), SCAN 4.2 software, and a modified 64-channel QuickCap with embedded $\mathrm{Ag} / \mathrm{AgCl}$ electrodes were used to record EEG signals from 62 active scalp sites referenced to vertex $(\mathrm{Cz})$ for the cap layout. The ground electrode was attached to the center of the forehead. The impedance was kept below $5 \mathrm{k} \Omega$. The EEG data are recorded with 16-bit quantization level at the sampling rate of 1000 Hz. Electrooculogram (EOG) was also recorded, and later used to identify blink artifacts from the recorded EEG data. 


\section{Feature Extraction}

The main task of feature extraction is to derive the salient features which can map the EEG data into consequent emotion states. For a comparison study, we investigated two different methods, one based on statistical features in the time domain, and the other based on power spectrum in the frequency domain. First, the EEG signals were down-sampled to a sampling rate of $200 \mathrm{~Hz}$ to reduce the burden of computation. Then, the time waves of the EEG data were visually checked. The recordings seriously contaminated by electromyogram (EMG) and Electrooculogram (EOG) were removed manually. Next, each channel of the EEG data was divided into 1000-point epochs with 400-point overlap. Finally, all features discussed below were computed on each epoch of all channels of the EEG data.

\subsection{Time-Domain Features}

In this paper, we use the following six different kinds of time-domain features [12.

a) The mean of the raw signal

$$
\mu_{X}=\frac{1}{N} \sum_{n=1}^{N} X(n)
$$

where $X(n)$ represents the value of the $n$th sample of the raw EEG signal, $n=1, \ldots N$.

b) The standard deviation of the raw signal

$$
\sigma_{X}=\left(\frac{1}{N-1} \sum_{n=1}^{N}\left(X(n)-\mu_{X}\right)^{2}\right)^{1 / 2}
$$

c) The mean of the absolute values of the first differences of the raw signal

$$
\delta_{X}=\frac{1}{N-1} \sum_{n=1}^{N-1}|X(n+1)-X(n)|
$$

d) The mean of the absolute values of the second differences of the raw signal

$$
\gamma_{X}=\frac{1}{N-2} \sum_{n=1}^{N-2}|X(n+2)-X(n)|
$$

e) the means of the absolute values of the first differences of the normalized signals

$$
\tilde{\delta}_{X}=\frac{1}{N} \sum_{n=1}^{N-1}|\tilde{X}(n+1)-\tilde{X}(n)|=\frac{\delta_{X}}{\sigma_{X}}
$$


where $\tilde{X}(n)=\frac{X(n)-\mu_{X}}{\sigma_{X}}, \mu_{X}$ and $\sigma_{X}$ are the means and standard deviations of $X$.

f) the means of the absolute values of the second difference of the normalized signals

$$
\tilde{\gamma}_{X}=\sum_{n=1}^{N-2}|\tilde{X}(n+2)-\tilde{X}(n)|=\frac{\gamma_{X}}{\sigma_{X}}
$$

\subsection{Frequency-Domain Features}

The frequency-domain features used in this paper are based on the power spectrum of each 1000-point EEG epochs. Analysis of changes in spectral power and phase can characterize the perturbations in the oscillatory dynamics of ongoing EEG. First, each epoch of the EEG data is processed with Hanning window. Then, windowed 1000-point epochs are further subdivided into several 200-point sub-windows using the Hanning window again with 100 point steps, and each is extended to 256 points by zero padding for a 256-point fast Fourier transform (FFT). Next, the power spectrum of all the sub-epochs within each epoch is averaged to minimize the artifacts of the EEG in all sub-windows. Finally, EEG log power spectrum are extracted in different bands such as delta rhythm, theta rhythm, alpha rhythm, beta rhythm, and gamma rhythm.

After these operations, we obtaine six kinds of time domain features and five kinds of frequency features. The dimension of each feature is 62 , and the number of each feature from each subject is about 1100 .

\section{Emotion Classification}

For an extensive evaluation of emotion recognition performance, classification of four emotional states is achieved by using three kinds of classifiers, $k \mathrm{NN}$ algorithm, MLPs, and SVMs. These classifiers have been separately applied to all of the aforementioned features. In this study, the Euclidean distance method is used as the distance metric for $k \mathrm{NN}$ algorithm. In MLPs, a three-layer neural network is adopted, and the activation function is the sigmoidal function. In SVMs, a radial basis function kernel is used.

In order to perform a more reliable classification process, we constructed a training set and a test set for each subject. The number of training set, which formed by the data of the former two sessions of each emotion, is about 700 for each subject. The number of test set, which formed by the data of the last session of each emotion, is about 400 for each subject.

Given the fact that a rather limited number of independent trials were available for each class, we apply cross-validation to select common parameters for each classifier, and pick the parameters that led to the highest average result in the training sets. For cross-validation, we chose a trial-based leave-oneout method (LOOM). In $k \mathrm{NN}$ training, we searched the number of neighbors 
Table 1. Classification accuracy using time domain features

\begin{tabular}{ccccccccc}
\hline Subject & Classifier & $\mu_{X}$ & $\sigma_{X}$ & $\delta_{X}$ & $\gamma_{X}$ & $\delta_{X}$ & $\tilde{\gamma}_{X}$ & All \\
\hline 1 & $k N N$ & 21.36 & 30.17 & 32.20 & 35.47 & 28.93 & 26.30 & 39.17 \\
& MLP & 23.15 & 29.32 & 34.38 & 36.23 & 29.71 & 29.47 & 40.21 \\
& SVM & 26.54 & 32.15 & 38.41 & 37.31 & 30.45 & 32.37 & $\mathbf{4 2 . 4 4}$ \\
\hline 2 & $k N N$ & 32.41 & 21.77 & 23.73 & 22.21 & 23.21 & 24.31 & 35.63 \\
& MLP & 33.58 & 23.51 & 25.39 & 24.17 & 26.42 & 28.90 & 37.40 \\
& SVM & 36.52 & 26.53 & 29.32 & 26.56 & 31.95 & 32.64 & $\mathbf{4 5 . 3 5}$ \\
\hline 3 & $k N N$ & 25.34 & 21.04 & 25.12 & 23.92 & 24.55 & 31.54 & 32.57 \\
& MLP & 26.73 & 22.25 & 27.35 & 26.84 & 26.78 & 33.35 & 35.79 \\
& SVM & 27.98 & 23.78 & 30.34 & 27.74 & 27.07 & 36.57 & $\mathbf{4 1 . 4 2}$ \\
\hline \multirow{4}{*}{4} & $k$ NN & 29.89 & 26.84 & 27.96 & 32.29 & 22.97 & 34.90 & 35.58 \\
& MLP & 28.25 & 26.86 & 29.08 & 33.32 & 24.62 & 35.52 & 38.07 \\
& SVM & 29.84 & 27.67 & 31.49 & 36.91 & 27.79 & 37.43 & $\mathbf{4 2 . 1 3}$ \\
\hline \multirow{5}{*}{5} & $k$ NN & 23.02 & 29.93 & 33.76 & 27.18 & 34.98 & 37.92 & 40.10 \\
& MLP & 26.91 & 28.47 & 35.82 & 30.85 & 36.26 & 39.76 & 41.98 \\
& SVM & 30.97 & 34.46 & 39.49 & 33.24 & 40.19 & 43.91 & $\mathbf{4 5 . 6 2}$ \\
\hline Average & $k$ NN & 26.40 & 25.95 & 28.57 & 28.21 & 26.93 & 30.99 & 36.61 \\
& MLP & 27.72 & 26.08 & 30.40 & 30.28 & 28.75 & 33.40 & 38.69 \\
& SVM & 30.37 & 28.92 & 32.95 & 32.35 & 31.49 & 36.58 & $\mathbf{4 3 . 3 9}$ \\
\hline
\end{tabular}

Table 2. Classification accuracy using frequency domain features

\begin{tabular}{cccccccc}
\hline \multicolumn{2}{c}{ Subject } & Classifier Delta & Theta & Alpha & Beta & Gamma & All frequcncy \\
\hline 1 & $k$ NN & 25.09 & 35.62 & 42.62 & 45.35 & 42.09 & 48.89 \\
& MLP & 26.91 & 36.36 & 45.13 & 47.64 & 44.45 & 51.18 \\
& SVM & 27.74 & 43.18 & 52.07 & 50.61 & 49.96 & $\mathbf{5 5 . 0 9}$ \\
\hline 2 & $k$ NN & 31.43 & 47.10 & 60.84 & 64.03 & 67.94 & 72.13 \\
& MLP & 40.51 & 54.62 & 61.83 & 63.90 & 70.74 & 80.67 \\
& SVM & 41.21 & 55.47 & 65.78 & 70.82 & 80.91 & $\mathbf{8 2 . 4 5}$ \\
\hline 3 & $k$ NN & 29.22 & 32.28 & 43.38 & 46.52 & 42.49 & 59.92 \\
& MLP & 35.35 & 45.84 & 46.42 & 49.69 & 47.28 & 61.34 \\
& SVM & 34.27 & 42.16 & 47.26 & 57.49 & 55.35 & $\mathbf{6 5 . 4 3}$ \\
\hline \multirow{2}{*}{4} & $k$ NN & 23.08 & 34.31 & 43.51 & 42.72 & 40.45 & 55.79 \\
& MLP & 27.38 & 36.52 & 45.02 & 45.82 & 42.37 & 57.91 \\
& SVM & 33.71 & 43.94 & 47.75 & 49.74 & 47.17 & $\mathbf{5 8 . 8 3}$ \\
\hline 5 & $k$ NN & 26.46 & 28.45 & 52.39 & 45.78 & 46.93 & 62.45 \\
& MLP & 28.17 & 30.48 & 53.20 & 46.57 & 45.09 & 64.26 \\
& SVM & 32.59 & 34.97 & 63.63 & 52.75 & 48.80 & $\mathbf{7 0 . 7 4}$ \\
\hline Average & $k$ NN & 27.05 & 35.55 & 48.54 & 48.88 & 47.98 & 59.84 \\
& MLP & 31.66 & 40.76 & 50.32 & 50.72 & 49.60 & 63.07 \\
& SVM & 33.90 & 43.94 & 55.29 & 56.28 & 56.43 & $\mathbf{6 6 . 5 1}$ \\
\hline
\end{tabular}


$k$. In MLP training, we searched the number of hidden neurons assigned to the MLPs. In SVM training, we searched the cost $C$ and $\gamma$ of the Gaussian kernel.

The experimental results of classification with different classifiers for statistical features in the time domain are given in Table 1. From this table, we can see that the classification performance of using all statistical features is evidently better than those based on individual features under the same conditions.

Table 2 shows the averaged classification performance of different classifiers using six frequency-domain features at different EEG frequency bands. From this table, we can see that the classification performance of using all frequency bands is evidently better than those based on individual frequency bands under the same condition. In addition, an interesting finding is implied that the frequency bands of alpha, beta, and gamma are more important than the frequency bands of delta and theta to the emotion classification.

From the results shown in Tables 1 and 2, we can see that the classification performance based on the frequency domain features were better than those based on time domain features. We can also found that the performance of SVM classifiers is better than those of $\mathrm{kNN}$ and MLPs, which proved true for all of the different features.

We tried to identify the significant features for each classification problem and thereby to investigate the class relevant feature domain and interrelation between the features for emotions. Feature extraction methods select or omit dimensions of the data that correspond to one EEG channel depending on a performance measure. Thus they seem particularly important not only to find the emotion-specific features but also expand the applicability of using fewer electrodes for practical applications. This study adopted minimum redundancymaximum relevance (MRMR), a method based on information theory for sorting each feature in descending order accounting for discrimination between different EEG patterns. Since the best performance was obtained using power spectrum across all frequencies, MRMR was further applied to this feature type to sort the feature across frequency bands.

Table 3 lists top-30 feature rankings of individual subject, which are obtained by applying MRMR to the training data set of each subject. The common features across different subjects are marked with a grey background. As we can see, the top-30 ranking features are variable for different subjects. Person-dependent differences of the EEG signals may account for the differences among different subjects 14. Moreover, every subject experiences emotions in a different way, which is probably another reason for this inter-subject variability. Nevertheless, there are still many similarities across different people. We can find that the features derived from the frontal and parietal lobes are used more frequently than other regions. This indicates that these electrodes provided more discriminative information than other sites, which is consistent with the neurophysiologic basis of the emotion 15 . 
Table 3. Top-30 feature selection results using MRMR

\begin{tabular}{|c|c|c|c|c|c|}
\hline & \multicolumn{5}{|c|}{ Subject } \\
\hline$\overline{\text { Rank }}$ & 1 & 2 & 3 & 4 & 5 \\
\hline 1 & AF4, Alpha & T7, Gamma & C6, Beta & F6, Beta & F8, Alpha \\
\hline 2 & C5, Beta & PZ, Delta & CP3, Theta & F8, Theta & O1, Gamma \\
\hline 3 & P3, Gamma & OZ, Alpha & F3, Gamma & TP8, Beta & FC3, Alpha \\
\hline 4 & FC3, Delta & T8, Gamma & F7, Alpha & FT7, Beta & C5, Theta \\
\hline 5 & T8, Alpha & C6, Delta & CP4, Delta & AF4, Alpha & F4, Alpha \\
\hline 6 & F1, Theta & TP8, Gamma & FT7, Beta & FC4, Delta & TP8, Theta \\
\hline 7 & C3, Theta & T8, Theta & P3, Alpha & C1, Gamma & T7, Gamma \\
\hline 8 & C4, Gamma & AF4, Alpha & CP5, Beta & FC3, Alpha & CP6, Beta \\
\hline 9 & F1, Gamma & F1, Gamma & POZ, Gamma & AF3, Delta & FC6, Gamma \\
\hline 10 & F3, Gamma & CP2, Theta & PO5, Gamma & CB1, Alpha & F8, Gamma \\
\hline 12 & T8, Beta & CP3, Theta & C4, Beta & F3, Gamma & CP2, Alpha \\
\hline 13 & AF3, Delta & CP6, Beta & FCZ, Theta & P6, Theta & C6, Delta \\
\hline 14 & F2, Beta & F3, Gamma & T7, Gamma & TP7, Theta & TP8, Beta \\
\hline 15 & FC5, Theta & FP2, Alpha & FO7, Alpha & C3, Theta & CP4, Gamma \\
\hline 16 & FC3, Theta & AF3, Delta & FCZ, beta & FT7, Theta & FP2, Alpha \\
\hline 17 & CP4, Beta & P2, Alpha & AF4, Alpha & F7, Gamma & F1, Delta \\
\hline 18 & CP6, Beta & C4, Theta & FC5, Alpha & F3, Delta & FCZ, Alpha \\
\hline 19 & T7, Gamma & FCZ, Alpha & C6, Delta & P3, Theta & OZ, Gamma \\
\hline 20 & FCZ, Alpha & C5, Gamma & F1, Delta & CP4, Gamma & AF3, Delta \\
\hline 21 & C4, Beta & OZ, Gamma & TP8, Beta & FP2, Alpha & CP5, Beta \\
\hline 22 & $\mathrm{P} 1$, Beta & PO8, Alpha & FC3, Theta & FZ, beta & AF4, Alpha \\
\hline 23 & TP8, Gamma & F8, Gamma & AF3, Delta & T7, Beta & P2, Alpha \\
\hline 24 & FP2, Alpha & PZ, Beta & FC1, Theta & FC3, Beta & C3, Theta \\
\hline 25 & CP5, Gamma & FP2, Gamma & C3, Theta & P6, Beta & C4, Beta \\
\hline 26 & CP4, Delta & CB2, Theta & FC2, Beta & O1, Alpha & FC3, Theta \\
\hline 27 & FT7, Beta & CPZ, Theta & FP2, Alpha & T7, Gamma & P3, Alpha \\
\hline 28 & PZ, Beta & C3, Theta & P2, Gamma & CP6, Beta & FC2, Beta \\
\hline 29 & TP8, Theta & CP3, Gamma & F4, Alpha & FC6, Alpha & F3, Gamma \\
\hline 30 & F7, Delta & CP4, Gamma & FC6, Gamma & FT8, Alpha & FC5, Theta \\
\hline
\end{tabular}

\section{Conclusion}

In this paper, we presented a study on EEG-based emotion recognition. Our experimental results indicate that it is feasible to identify four emotional states, joy, relax, fear and sad, during watching movie, and an average test accuracy of $66.51 \%$ is obtained by combining EEG frequency domain features and support vector machine classifiers. In addition, the experimental results show that the frontal and parietal EEG signals were more informative about the emotional states.

Acknowledgments. This research was supported in part by the National Natural Science Foundation of China (Grant No. 90820018), the National Basic 
Research Program of China (Grant No. 2009CB320901), and the European Union Seventh Framework Program (Grant No. 247619).

\section{References}

1. Picard, R.: Affective computing. The MIT press (2000)

2. Petrushin, V.: Emotion in speech: Recognition and application to call centers. Artificial Neu. Net. In Engr., 7-10 (1999)

3. Black, M., Yacoob, Y.: Recognizing facial expressions in image sequences using local parameterized models of image motion. International Journal of Computer Vision 25(1), 23-48 (1997)

4. Kim, K., Bang, S., Kim, S.: Emotion recognition system using short-term monitoring of physiological signals. Medical and Biological Engineering and Computing 42(3), 419-427 (2004)

5. Brosschot, J., Thayer, J.: Heart rate response is longer after negative emotions than after positive emotions. International Journal of Psychophysiology 50(3), 181-187 (2003)

6. Chanel, G., Kronegg, J., Grandjean, D., Pun, T.: Emotion assessment: Arousal evaluation using eegs and peripheral physiological signals. Multimedia Content Representation, Classification and Security, 530-537 (2006)

7. Davidson, R., Fox, N.: Asymmetrical brain activity discriminates between positive and negative affective stimuli in human infants. Science 218(4578), 1235 (1982)

8. Davidson, R., Schwartz, G., Saron, C., Bennett, J., Goleman, D.: Frontal versus parietal eeg asymmetry during positive and negative affect. Psychophysiology 16(2), 202-203 (1979)

9. Bos, D.: Eeg-based emotion recognition. The Influence of Visual and Auditory Stimuli

10. Takahashi, K.: Remarks on emotion recognition from bio-potential signals. In: The Second International Conference on Autonomous Robots and Agents, pp. 667-670. Citeseer (2004)

11. Nie, D., Wang, X.W., Shi, L.C., Lu, B.L.: EEG-based emotion recognition during watching movies. In: The Fifth International IEEE/EMBS Conference on Neural Engineering, pp. 186-191. IEEE Press, Mexico (2011)

12. Bradley, M., Lang, P.: Measuring emotion: the self-assessment manikin and the semantic differential. Journal of Behavior Therapy and Experimental Psychiatry 25(1), 49-59 (1994)

13. Picard, R.W., Vyzas, E., Healey, J.: Toward machine emotional intelligence: Analysis of affective physiological state. IEEE Transactions on Pattern Analysis and Machine Intelligence 23(10), 1175-1191 (2001)

14. Heller, W.: Neuropsychological mechanisms of individual differences in emotion, personality, and arousal. Neuropsychology 7(4), 476 (1993)

15. Schmidt, L., Trainor, L.: Frontal brain electrical activity (eeg) distinguishes valence and intensity of musical emotions. Cognition Emotion 15(4), 487-500 (2001) 